\title{
INDUCTION SYSTEM FOR A PROTON BUNCH ACCELERATION IN SYNCHROTRON *
}

\author{
Kota Torikai, Yoshio Arakida, Eiji Nakamura, Tadaaki Kono, Yoshito Shimosaki, Takeshi Toyama, \\ Masayoshi Wake, Junichi Kishiro and Ken Takayama
}

KEK, Ibaraki Oho 1-1, Tsukuba, Japan

\begin{abstract}
Recently the first induction acceleration of a single proton bunch of 2.3E10ppp was demonstrated [1], where it was accelerated from $500 \mathrm{MeV}$ to $8 \mathrm{GeV}$ in $1.9 \mathrm{sec}$ in KEK-PS. This paper describes the induction system for a synchrotron and a beam confinement experiment as a second step of the proof-of principle ( $\mathrm{PoP}$ ) experiments of a "induction synchrotron" [2], where a proton beam with a bunch length of 500ns was longitudinally confined by the induction barriers for $500 \mathrm{msec}$, is introduced..
\end{abstract}

\section{INDUCTION SYSTEM FOR SYNCHROTRON}

The induction system required for the proton bunch acceleration was integrated in September 2004. The installed components are: 1) the switching power supplies $[3], 2)$ the induction cells [4], 3) the trigger system, and 4) the interlock system. The entire system is described in an equivalent circuit, shown Figure 1.

\section{Switching Power Supply}

A DC power supply, which is connected to the switching power supply (SPS) via a power cable of $100 \mathrm{~m}$, transforms the input voltage of $\mathrm{AC} 400 \mathrm{~V}$ to a maximum output voltage of $3.0 \mathrm{kV}$ by switching IGBT.

The switching power supply is formed from a fullbridge switching circuit. Each switching arm consists of 7 series of MOS-FET, so as to withstand a maximum voltage of $2.5 \mathrm{kV}$ with an arm current of $18 \mathrm{~A}$. A rise-time and fall-time of the voltage pulse is 30ns each. A maximum repetition rate of the SPS is limited by a temperature rise of the MOS-FETs due to switching loss, which is estimated to be $150 \mathrm{~W} / 3.7 \mathrm{~cm}^{2}$. An indirect watercooling copper block is contacted to a surface of the MOS-FETs. A total heat deposit of $4.2 \mathrm{~kW}$ is removed by a water flow of $12 \mathrm{~L} / \mathrm{min}$. Three SPSs, shown in Figure 2, are placed $40 \mathrm{~m}$ away from the accelerator in order to avoid radiation damages on MOS-FETs. The pulse power is transmitted to the induction cell through the $40 \mathrm{~m}$ transmission cable of $125 \mathrm{ohm}$.

Gate signals of the MOS-FETs are transferred from the trigger system, which is described below, in the form of optical signal. The gate terminal of the each MOS-FET is insulated from a ground. An electric power, which is required for driving the gate of the MOS-FET, is supplied from DC-DC converters. An input terminal of the SPS is a group of film condensers to keep the output voltage from a perturbing change in the charged voltage.

\section{Induction Cell}

One induction cell consists of 6 magnetic cores, which are made of nanocrystalline alloy (FINEMET). Each magnetic core has an outer diameter of $500 \mathrm{~mm}$, inner diameter of $225 \mathrm{~mm}$, and thickness of $15 \mathrm{~mm}$. A diameter of a induction cell beam pipe is $150 \mathrm{~mm}$. A ceramic gap of $5 \mathrm{~cm}$ in length and $8 \mathrm{~mm}$ in thickness is employed. Silicone oil, which is helpful to decrease a stray capacitance, is a coolant for the induction cell. A flowrate of the coolant of the induction cell is $70 \mathrm{~L} / \mathrm{min}$, providing a maximum cooling capacity of $20 \mathrm{~kW}$. The induction cells aligned on the beam line are shown in Figure 2.

An equivalent electric circuit of the induction cell is represented as a parallel connection of an inductance of $110 \mu \mathrm{H}$, a capacitance of $280 \mathrm{pF}$, and a resistance of $300 \mathrm{ohm}$. These numbers have been obtained from a fitting result of a measurement of a beam-induced voltage of the induction cell [4].

A matching resistor of $210 \mathrm{ohm}$, which is determined so as to improve the impedance matching to the transmission cable around the revolution frequency of $667 \mathrm{kHz}-$ $882 \mathrm{kHz}$, is connected in parallel to the induction cell. A peak impedance of the (induction cell + matching resistor) is $141 \mathrm{ohm}$ at $5.9 \mathrm{MHz}$, and an obtained quality factor of the load is 0.1 . The matching load is indirectly water-cooled. A flow-rate of the cooling water is $5 \mathrm{~L} / \mathrm{min}$, providing a maximum cooling capacity of $15 \mathrm{~kW}$. The single induction cell is capable of generating an acceleration voltage of $2 \mathrm{kV}$. Three cells have been installed for the first POP experiment.

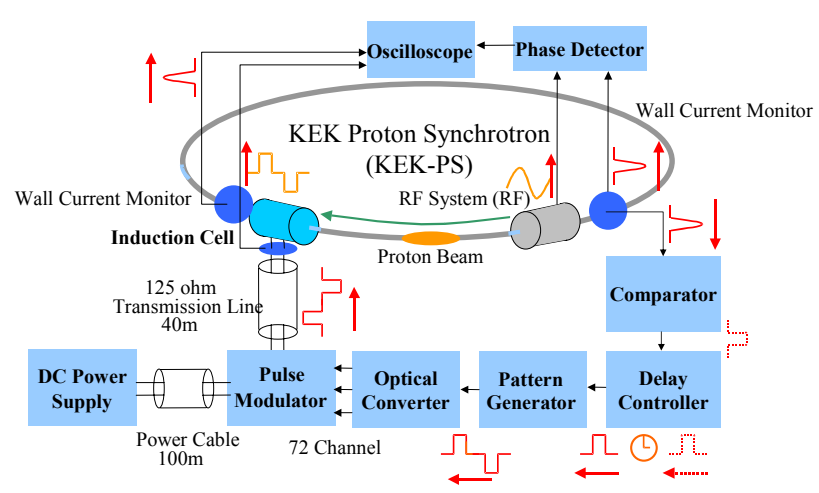

Figure 1: Signal Flow Diagram of Induction System 

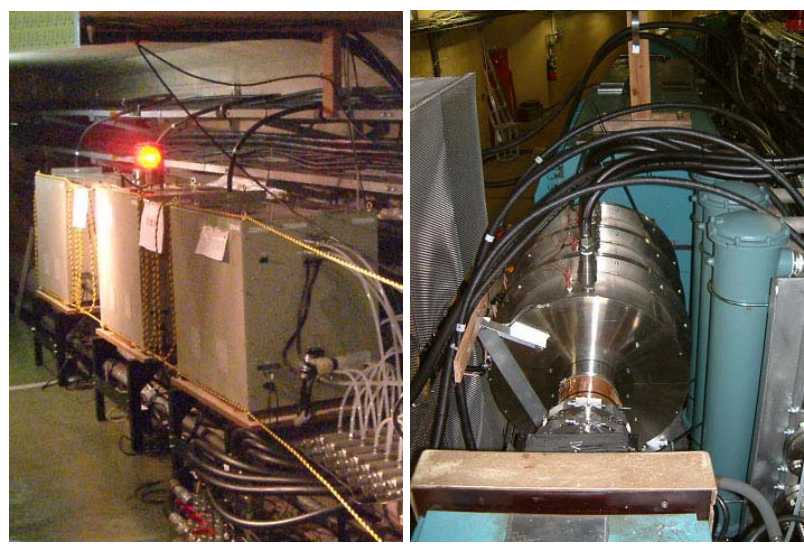

Figure 2: Switching Power Supplies (left) and Induction Cells with Matching Load (right)

\section{Trigger System}

The trigger system consists of four electric components: 1) voltage comparator, 2) delay module, 3 ) pulse-pattern generator, and 4) electric/optical signal converter. Signal flows in the trigger system are shown in Figure 1. The wall current monitor (WCM) generates an initial trigger signal. A rising edge of the WCM signal is detected by the voltage comparator in the accelerator central control room (CCR), and a timing signal of rectangular shape are generated. Subsequently the timing signal is transferred to the delay module, in order to keep the generation of the induction voltage synchronized with the beam passing through the induction cell. Then, the delayed signal is transferred to the pulse-pattern generator. A pulse width of the induction voltages can be varied by changing on/off timing of the switching arm in the SPS. A delayed negative voltage to a positive induction voltage can be controlled in a similar way. Basically the pulse width of the positive / negative voltage must be same, in order to avoid input of an equivalent $\mathrm{DC}$ voltage.

The pulse pattern signal is transferred to the electric/optical signal converter placed near the SPS through the $80 \mathrm{~m}-\log$ transmission cables of $50 \mathrm{ohm}$. The converter and the SPS are connected by the $10 \mathrm{~m}$-long optical fiber cable. These optical signals serve to drive MOS-FETs as their gate signals.

\section{Interlock System}

Each component of the induction system is endowed with an interlock system to protect itself from any malfunction. A programmable logic controller (PLC) is used to manage interlock signals. The interlock system detects fatal troubles, such as a temperature rise in the induction cell, insufficient oil flow, insufficiency water flow in the SPS/the matching resistor, and overvoltage/over-current in the MOSFETs.

In a case of safety, an input of an enable signal from the interlock system is required in order to output trigger signal. And a manual input of a reset pulse to the interlock system is required in order to resume from the trouble.

\section{BEAM-RF PHASE MEASUREMENT}

A measurement of the bunch position in the rf-phase, which is monitored by the phase detector integrated in the rf-feedback control system through the entire stage of acceleration, is indispensable to experimentally confirm the induction acceleration of a proton bunch in the hybrid system, where the induction acceleration system is combined with the rf system. When the beam acceleration is ideal, the bunch should stay on a specific phase of an rf, $\phi_{s}=\sin ^{-1}\left[\rho C_{0}(d B / d t) / V_{r f}\right]$, where $\rho, C_{0}, B$, and $V_{r f}$ are the bending radius, circumference of an accelerator ring, bending field, and amplitude of the rf, respectively. An actually obtained output-signal of the phase detector $V_{\text {out }}$, which is closely related to $\phi_{s}$, is affected by a finite gain factor and off-set. Therefore a calibration of the phase detector has been carried out by the practical method of varying the rf amplitude and observing the output-signal of the phase detector.

Assuming a temporal evolution of a linear gain factor $a(t)$ and off-set term $b(t), V_{\text {out }}\left(t, \varphi_{s}\left(V_{r f}\right)\right)$ is written by $V_{\text {out }}\left(t, \phi_{s}\left(V_{r f}\right)\right)=a(t) \phi_{s}\left(V_{r f}\right)+b(t)$. By varying $V_{r f}$, data array of the theoretically estimated $\varphi_{s}\left(V_{r f}\right)$ and $V_{\text {out }}$ at a certain instant $t$ of the acceleration period can be obtained as is shown in Figure 3. In this figure $t=500 \mathrm{~ms}$ for an example. Finally $a(t)$ and $b(t)$ is obtained by applying least-squares method to these plots at each instant, which is shown in Figure 4.

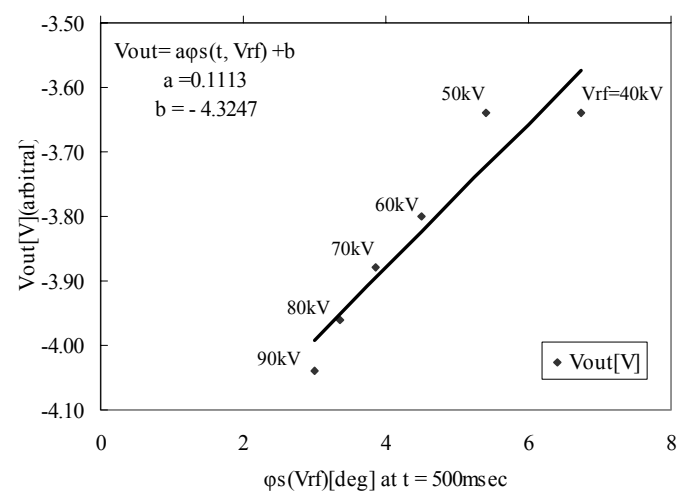

Figure 3: An Example of $\Delta \varphi$ signal at $t=500 \mathrm{msec}$

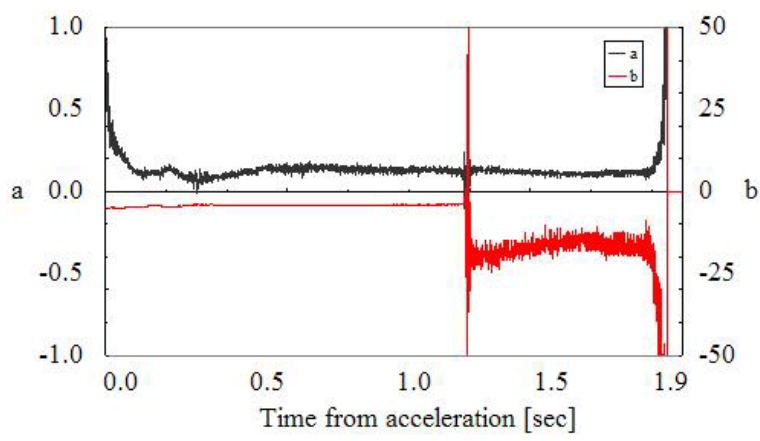

Figure 4: Temporal evolution of the linear gain factor $a(t)$ and offset term $b(t)$

Theoretically estimated accelerating phases for different Vrf are given in Table 1. And the temporal evolution of a 
calibrated output signal of the phase monitor by $\mathrm{rf}$ acceleration is shown in Figure 5. A comparison of the required accelerating phase and the calibrated output signal indicates that the output signal of the phase detector is valid for measuring the acceleration phase.

Table 1 Estimated Acceleration Phase

\begin{tabular}{|c|c|c|c|c|c|c|}
\hline $\mathrm{rf}[\mathrm{kV}]$ & 40 & 50 & 60 & 70 & 80 & 90 \\
\hline$\Delta \varphi[\mathrm{deg}]$ & 6.7 & 5.4 & 4.5 & 3.8 & 3.3 & 3.0 \\
\hline
\end{tabular}
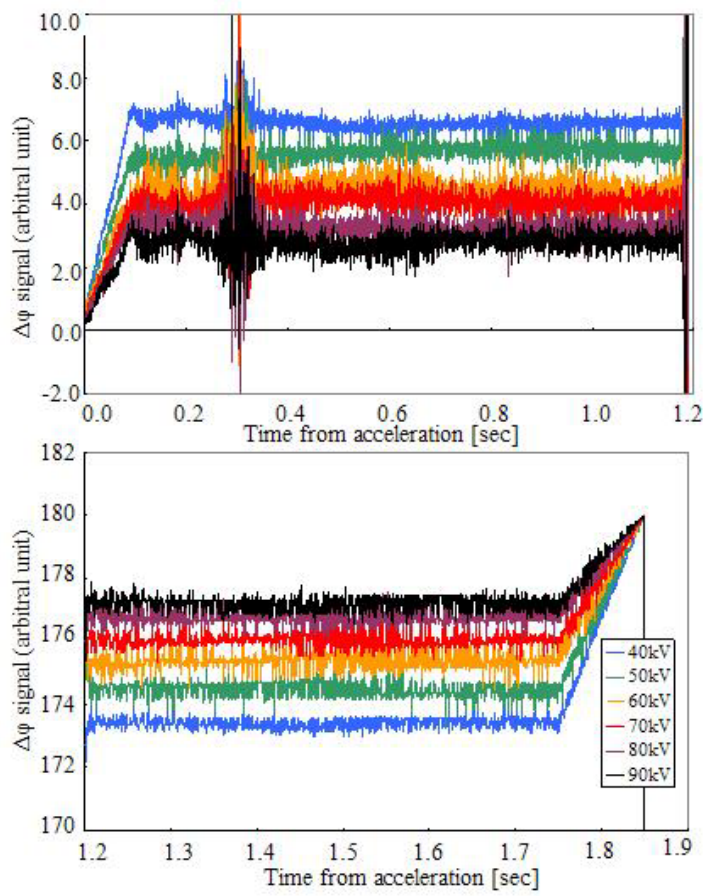

Figure 5: Calibrated Phase Data of RF Acceleration

(Before / After Transition: Upper / Lower)

\section{BEAM CONFINEMENT BY INDUCTION VOLTAGE}

The beam confinement using the induction step barriers is crucial to achieve a super-bunch for future applications [5]. A 5.0E10 ppp of proton beam was successfully confined in a shallow notch potential generated by the induction voltage $V_{\text {barrier }}$ of $4.7 \mathrm{kV}$, with pulse width $T_{1}$ of $400 \mathrm{~ns}$. An outline of the experiment is shown in Figure 6.

Assuming a rectangular barrier voltage, the bucket height in energy $\Delta E_{\max }$ is expressed in terms of the pulse voltage $V_{\text {barrier }}$, a pulse width $T_{1}$, a revolution period $T_{0}$, a velocity of the reference particle $\beta$, energy of the synchronous particle $E_{0}$, and a slippage factor $\eta$,

$$
\Delta E_{\max }=\sqrt{\frac{e V_{\text {barrier }} T_{1}}{T_{0}} \frac{2 \beta^{2} E_{0}}{|\eta|}} .
$$

This experiment was carried out at the injection energy $(500 \mathrm{MeV})$ of the KEK-PS, where $T_{0}=1.51 \mu \mathrm{s}, \beta=0.751$, $E_{0}=(500+938) \mathrm{MeV}$, and $\Delta E_{\max }=0.18 \%$. An experimental result is shown in Figure 7, where a plotted line from bottom to top in the graph represents a time evolution of the beam shape in a longitudinal direction. In this experiment, $1.0 \mathrm{E} 11 \mathrm{ppp}$ of proton beam with maximum energy spread of $0.15 \%$ is injected. From a comparison of the energy spread of the beam and the depth of barrier potential, all injected particle was to be confined within the barrier voltage. However, $50 \%$ of injected beam was lost during $460 \mathrm{~ms}$. Possible causes of the beam loss are under investigation.

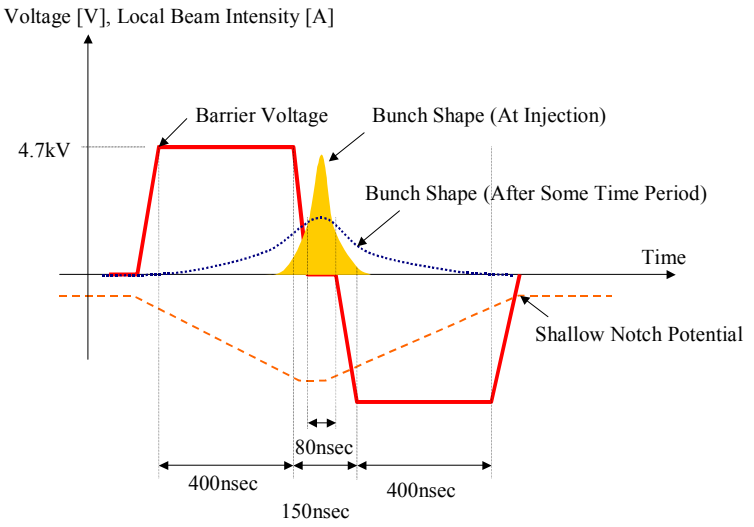

Figure 6: Beam Confinement by Induction Voltage

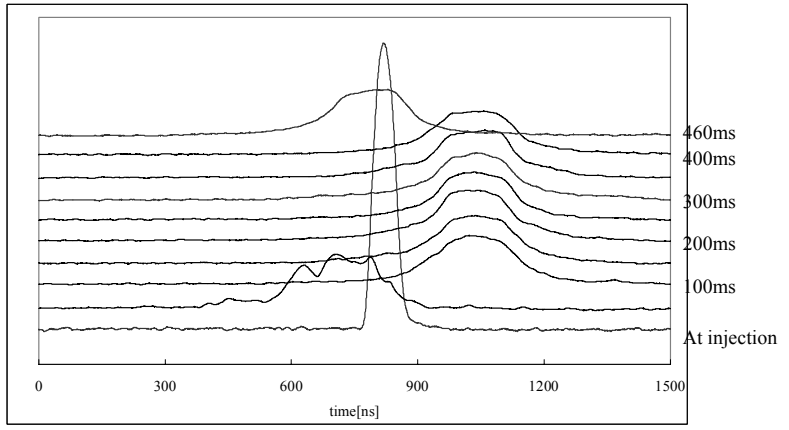

Figure 7: Bunch Shape during Experiment of Beam confinement

\section{REFERENCES}

[1] Ken Takayama et. al., Phys. Rev. Lett. 94, 144801 (2005).

[2] K.Takayama and J.Kishiro, Nucl. Inst. Meth. A 451, 304 (2000).

[3] Kunio Koseki, PhD Thesis (Sokendai), to be published.

[4] Kota Torikai et. al., , "Design Study of $1 \mathrm{MHz}$ Induction Cavity For Induction Synchrotron", in proceedings of PAC2003, pp.1784-1786 (2004).

[5] K.Takayama et al., Phys. Rev. Lett. 88, 144801 (2002). 